\title{
Violations of Einstein's Relativity: Motivations, Theory, and Phenomenology
}

\author{
Ralf Lehnert \\ Instituto de Ciencias Nucleares, Universidad Nacional Autónoma de México, A. Postal 70-543, 04510 México D.F., Mexico
}

(Dated: February 16, 2011)

\begin{abstract}
One of the most difficult questions in present-day physics concerns a fundamental theory of space, time, and matter that incorporates a consistent quantum description of gravity. There are various theoretical approaches to such a quantum-gravity theory. Nevertheless, experimental progress is hampered in this research field because many models predict deviations from established physics that are suppressed by some power of the Planck scale, which currently appears to be immeasurably small.

However, tests of relativity theory provide one promising avenue to overcome this phenomenological obstacle: many models for underlying physics can accommodate a small breakdown of Lorentz symmetry, and numerous feasible Lorentz-symmetry tests have Planck reach. Such mild violations of Einstein's relativity have therefore become a focus of recent research efforts.

This presentation provides a brief survey of the key ideas in this research field and is geared at both experimentalists and theorists. In particular, several theoretical mechanisms leading to deviations from relativity theory are presented; the standard theoretical framework for relativity violations at currently accessible energy scales (i.e., the SME) is reviewed, and various present and near-future experimental efforts within this field are discussed.

PACS numbers: 11.30.Cp, 12.20.-m, 41.60.Bq, 29.20.-c
\end{abstract}

\section{INTRODUCTION}

Despite their phenomenological success, the Standard Model of particle physics and general relativity leave unresolved a number of theoretical questions. For this reason, a considerable amount of theoretical effort is currently directed toward the search for a more fundamental theory that includes a quantum description of the gravitational field. However, experimental tests of theoretical approaches to a quantum theory of gravity face a key issue of practical nature: most quantum-gravity effects in virtually all leading candidate models are expected to be minuscule as a result of Planck-scale suppression. For instance, measurements at presently attainable energies are likely to demand sensitivities at the $\sim 10^{-17}$ level or better. This mini course gives an overview of one recent approach to this issue involving the violation of spacetime symmetries.

Due to the expected minute size of candidate quantum-gravity effects, promising experimental avenues are difficult to identify. One idea in this context is testing physical laws that obey three key criteria. The first criterion is that one should focus on fundamental laws that hold exactly in established physical theories at the fundamental level. Any observed deviations from these laws would then definitely imply qualitatively new physics. Second, the likelihood of measuring such deviations is increased by testing laws that are predicted to be violated in credible approaches to more fundamental physics. The third criterion is a practical one: for the potential to detect Planck-suppressed effects, these laws should be amenable to ultrahigh-precision tests.

One sample physics law that satisfies all of these criteria is CPT symmetry [1. As a brief reminder, this fundamental law states that all physics remains invariant under the combined operations of charge conjugation (C), parity inversion $(\mathrm{P})$, and time reversal $(\mathrm{T})$. Here, the $\mathrm{C}$ transformation links particles and antiparticles, $\mathrm{P}$ denotes the spatial reflection of physics quantities through the coordinate origin, and T reverses a given physical process in time. The Standard Model of particle physics is CPT symmetric by construction, so that the first criterion is satisfied. In the context of criterion two, we mention that a variety of candidate fundamental theories can accomodate CPT violation. Such approaches include string theory 2, spacetime foam 3, nontrivial spacetime topology 4, and cosmologically varying scalars [5]. The third criterion above is met as well. Consider, for example, the conventional figure of merit for CPT symmetry in the neutral-Kaon system: its value lies presently at $10^{-18}$, as quoted by the Particle Data Group [6].

Similar arguments can also be made for other spacetime symmetries, such as Lorentz and translational symmetry. They are ingrained into our current understanding of physics at the fundamental level; they can be affected in various quantum-gravity approaches because quantum gravity is likely to require a radically different "spacetime" concept at the Planck length; and being symmetries, ultrahigh-senstivity searches for deviations from Lorentz and translational invariance can be devised. The point is that tests of discrete and continuous spacetime symmetries have become a key tool in the phenomenology of new physics that possibly arises at the Planck scale.

This mini course is organized as follows. Section [II discusses the relation between various spacetime symmetries. Two sample mechanisms for CPT- and Lorentz-symmetry violation in Lorentz-invariant underlying theories are reviewed in Sec. III The basic philosophy and ideas behind the construction of the Standard-Model Extension (SME) 
are presented in Sec. IV Section V comments on a number of Lorentz and CPT tests in a variety of physical systems. A brief summary is contained in Sec. VI.

\section{THE INTERPLAY BETWEEN DIFFERENT SPACETIME SYMMETRIES}

Spacetime transformations can be divided into two distinct sets, namely discrete and continuous transformations. The discrete transformations include $\mathrm{C}, \mathrm{P}$, and $\mathrm{T}$ discussed in the introduction, as well as various combinations of these, such as CP and CPT. Examples of possible continuous transformations are translations, rotations, and boosts. If symmetry under one or more of these transformations is lost, it is natural to ask as to whether the remaining transformations continue to be symmetries, or if the violation of one type of spacetime symmetry can lead to the breakdown of other spacetime invariances. This sections contains a few remarks about this issue.

We begin by considering the CPT transformation. The renowned CPT theorem, established by Bell, Lüders, and Pauli, essentially states the following: under a few mild assumptions, symmetry under CPT is a consequence of quantum theory, locality, and Lorentz invariance. If deviations from CPT symmetry were observed in nature, one or more of the ingredients necessary to prove the CPT theorem must be incorrect. The question now becomes which one of the key ingredients that enter the CPT theorem should be dropped.

The answer depends largely on the presumed underlying physics. But suppose the low-energy leading-order effects of new physics can be described within a local effective field theory. (Effective field theory is an enormously flexible tool. In the past, it has been successfully applied in numerous contexts including condensed-matter systems, nuclear physics, and elementary-particle physics.) It then seems unavoidable that exact Lorentz symmetry needs to be abandoned. This expectation has recently been proven rigorously in the context of axiomatic quantum field theory by Greenberg. His "anti-CPT theorem" roughly states that in any unitary, local, relativistic point-particle field theory CPT violation comes with Lorentz breakdown [7, 8. However, it is important to note that the converse of this statement-namely that Lorentz violation implies CPT breakdown - does not hold true in general. In any case, we see that in the above general and plausible context, CPT tests also probe Lorentz invariance. We remark that other types of CPT violation arising from apparently non-unitary quantum mechanics have also been considered [9].

We continue by supposing that translational invariance is violated. This possibility can arise in the context of cosmologically varying scalar fields (see next section). When translational symmetry is lost, the generator of translations (i.e., the energy-momentum tensor $\theta^{\mu \nu}$ ) is typically no longer a conserved current. We now turn to the question as to whether Lorentz symmetry would be affected in such a scenario. We begin by looking at the generator for Lorentz transformations, the angular-momentum tensor $J^{\mu \nu}$, which is given by

$$
J^{\mu \nu}=\int d^{3} x\left(\theta^{0 \mu} x^{\nu}-\theta^{0 \nu} x^{\mu}\right) .
$$

Note that this definition contains the energy-momentum tensor $\theta^{\mu \nu}$, which is not conserved in the present context. As a result, $J^{\mu \nu}$ will generally exhibit a nontrivial dependence on time, so that the ordinary time-independent Lorentztransformation generators no longer exist. For this reason, exact Lorentz symmetry is not guaranteed. It is apparent that (with the exception of special cases) translation-symmetry breaking leads to Lorentz-invariance violation.

\section{SAMPLE MECHANISMS FOR SPACETIME-SYMMETRY BREAKING}

In the previous section, we have argued that under certain circumstances the breakdown of one spacetime invariance may lead to the violation of another spacetime symmetry. Perhaps a more interesting question is how a translation-, Lorentz-, and CPT-invariant underlying model can lead to the breakdown of a particular spacetime symmetry in the first place. The present section addresses this issue by giving some intuition regarding mechanisms for spacetimesymmetry violation in candidate fundamental theories. Of the various possibilities for Lorentz breakdown mentioned in the introduction, we will focus on spontaneous Lorentz and CPT violation as well as Lorentz and CPT breaking through cosmologically varying scalars.

Spontaneous Lorentz and CPT breakdown. The mechanism of spontaneous symmetry breaking is well established in many areas of physics including the physics of elastic media, condensed-matter physics, and elementaryparticle theory. From a theoretical perspective, this mechanism is very appealing for the following reason. Often, a symmetry is needed for the internal consistency of a quantum field theory, but the symmetry is not observed in nature. This is exactly what spontaneous symmetry breaking achieves: At the dynamical level, the symmetry remains intact and ensures consistency. It is only the ground-state solution (which pertains to experiments) that is associated with the loss of the symmetry. In order to gain intuition about the spontaneous breaking of Lorentz and CPT symmetry, 
we will look at three sample physical systems whose features will lead us step by step to a better understanding of the effect. These three examples are illustrated in Fig. 1.

Let us first consider classical electrodynamics. Any electromagnetic-field configuration possesses an energy density $V(\vec{E}, \vec{B})$, which is determined by

$$
V(\vec{E}, \vec{B})=\frac{1}{2}\left(\vec{E}^{2}+\vec{B}^{2}\right) .
$$

Here, natural units have been implemented, and $\vec{E}$ and $\vec{B}$ denote the electric and magnetic field, respectively. Equation (2) yields the field energy of any given solution of the usual Maxwell equations. Notice that if the electric field, or the magnetic field, or both are different from zero in some region of spacetime, then the energy stored in these fields will be strictly positive. The field energy only vanishes when both $\vec{E}$ and $\vec{B}$ are zero throughout spacetime. The vacuum is usually identified with the ground state, which is the lowest-energy configuration of a system. It is thus apparent that in conventional electrodynamics the configuration with the lowest energy is the field-free one, so that the Maxwell vacuum is empty (disregarding Lorentz- and CPT-symmetric zero-point quantum fluctuations).

Let us next consider a Higgs-type field. Such a field is contained in the phenomenologically very successful Standard Model of particle physics. As opposed to the electromagnetic field, which is a vector, the Higgs field is a scalar. In our example, we will simplify various aspects of the model without distorting the features relevant in the present context. In the case for a constant Higgs-type field $\varphi$, the expression for the energy density of $\varphi$ is given by

$$
V(\varphi)=g\left(\varphi^{2}-\lambda^{2}\right)^{2}
$$

where $\lambda$ and $g$ are constants. (A possible spacetime dependence $\varphi=\varphi(x)$ would lead to additional, positive-valued contributions to the energy density, so we can indeed focus on constant $\varphi$.) Paralleling the electrodynamics case described above, the lowest possible energy of $\varphi$ is zero. However, in contrast to the Maxwell example this lowestenergy configuration requires $\varphi$ to be nonzero: $\varphi= \pm \lambda$. As a result, the physical low-energy vacuum for a system involving a Higgs-type field $\varphi$ is not empty; it contains, in fact, the spacetime-constant scalar field $\varphi_{v a c} \equiv\langle\varphi\rangle= \pm \lambda$. Here, the quantity $\langle\varphi\rangle$ denotes the vacuum expectation value (VEV) of $\varphi$. We remark in passing that a key physical effects of the VEV of the Standard-Model Higgs is to generate masses for many elementary particles. It is important to note that $\langle\varphi\rangle$ is a scalar, and therefore it does not select a preferred direction in spacetime.

Our final example is a 3 -vector field $\vec{R}$. (The relativistic generalization to 4-vectors or 4-tensors is straightforward.) This field (or its generalization) is not contained in the Standard Model, and there is no observational evidence for such a field at the present time. However, additional vector fields like $\vec{R}$ are present in numerous candidate fundamental theories. Paralleling the Higgs case, we take the expression for the energy density of $\vec{R}=$ const. to be

$$
V(\vec{R})=\left(\vec{R}^{2}-\lambda^{2}\right)^{2}
$$

It is apparent that the lowest possible energy is zero, just as in the previous two examples involving electromagnetism and a Higgs-type scalar. As for the Higgs, this lowest-energy configuration necessitates a nonzero $\vec{R}$. In particular, we must require that $\vec{R}_{v a c} \equiv\langle\vec{R}\rangle=\vec{\lambda}$, where $\vec{\lambda}$ is any constant vector obeying $\vec{\lambda}^{2}=\lambda^{2}$. As in the Higgs case, the vacuum does not stay empty; it rather contains the VEV of the vector field, $\langle\vec{R}\rangle$. Since we have only taken spacetime-independent solutions $\vec{R}$ into consideration, $\langle\vec{R}\rangle$ is also constant. (A possible $x$ dependence would lead to positive definite derivative terms in Eq. (4) raising the energy density, as in the other two of the above examples.) Hence, the true vacuum in the above model exhibits an intrinsic direction given by $\langle\vec{R}\rangle$. The upshot is that such an intrinsic direction violates rotation invariance and thus Lorentz symmetry. We note that interactions generating energy densities like those in Eq. (4) are absent in conventional renormalizable gauge theories, but they can be found in the context of string field theory, for instance.

Spacetime-varying scalars. A spacetime-dependent scalar, regardless of the mechanism causing this dependence, typically leads to the breakdown of spacetime-translation invariance [5]. In Sec. II] we have argued that translations and Lorentz transformations are closely linked in the Poincaré group, so that translation-symmetry violation typically leads to Lorentz breakdown. In the remainder of this section, we will focus on an explicit example for this effect.

Consider a system with a spacetime-dependent coupling $\xi(x)$ and scalar fields $\phi$ and $\Phi$, and take the Lagrangian $\mathcal{L}$ to contain a kinetic-type term $\xi(x) \partial^{\mu} \phi \partial_{\mu} \Phi$. Under mild assumptions, one may integrate by parts the action for this system (for instance with respect to the first partial derivative in the above term) without modifying the equations of motion. An equivalent Lagrangian $\mathcal{L}^{\prime}$ would then be given by

$$
\mathcal{L}^{\prime} \supset-K^{\mu} \phi \partial_{\mu} \Phi
$$


(a)

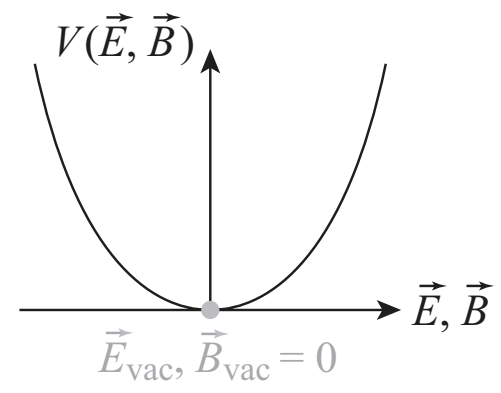

(b)

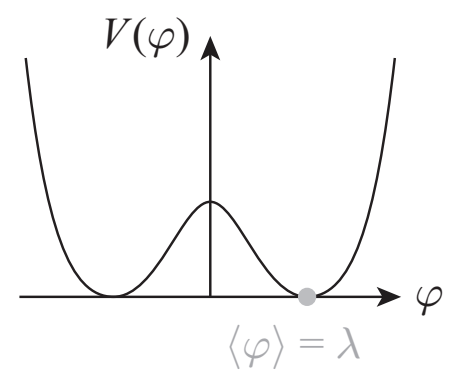

(c)

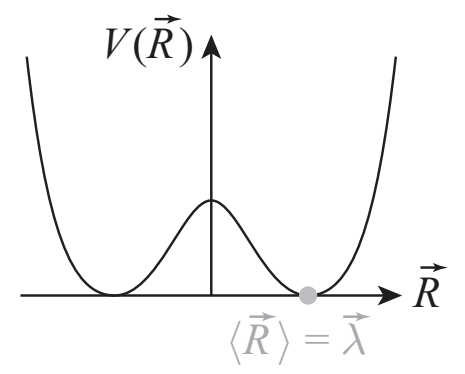

empty vacuum

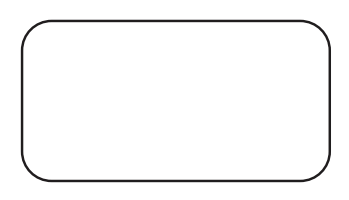

vacuum with scalar condensate

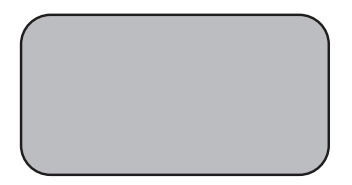

vacuum with vector condensate

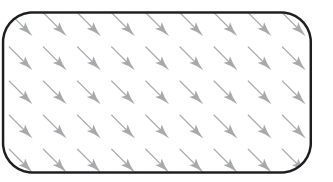

FIG. 1: Spontaneous symmetry breaking. In ordinary electrodynamics (a), the lowest-energy configuration is attained for zero $\vec{E}$ and $\vec{B}$ fields. The vacuum stays essentially free of fields. For a Higgs-type field (b), interactions generate an energy density $V(\varphi)$ that requires a non-vanishing value of $\varphi$ in the ground state. The vacuum contains a scalar condensate depicted in gray. Lorentz and CPT symmetry still hold (but other, internal symmetries may be broken). Vector fields present in string theory (c), for example, can possess interactions similar to those of the Higgs demanding a nonzero field value in the lowest-energy state. The VEV of a vector field would select a preferred direction in the vacuum, which breaks Lorentz and possibly CPT invariance.

where $K^{\mu}(x) \equiv \partial^{\mu} \xi(x)$ is an external prescribed 4-vector. This 4-vector clearly selects a preferred direction in spacetime breaking Lorentz invariance. We remark that for variations of $\xi(x)$ on cosmological scales, $K^{\mu}$ is spacetime constant locally (say on solar-system scales) to an excellent approximation.

The breakdown of Lorentz symmetry in the presence of a varying scalar can be understood intuitively as follows. The 4-gradient of the scalar has to be nonzero in some spacetime regions, for otherwise the scalar would be constant. This 4-gradient then singles out a preferred direction in such regions, as is illustrated in Fig. 2. Consider, for example, a particle that possesses certain interactions with the scalar. Its propagation properties might be affected differently in the directions perpendicular and parallel to the gradient. But physically inequivalent directions are associated with rotation-symmetry breaking. Since rotations are contained in the Lorentz group, Lorentz invariance must be violated. 
vacuum with varying scalar

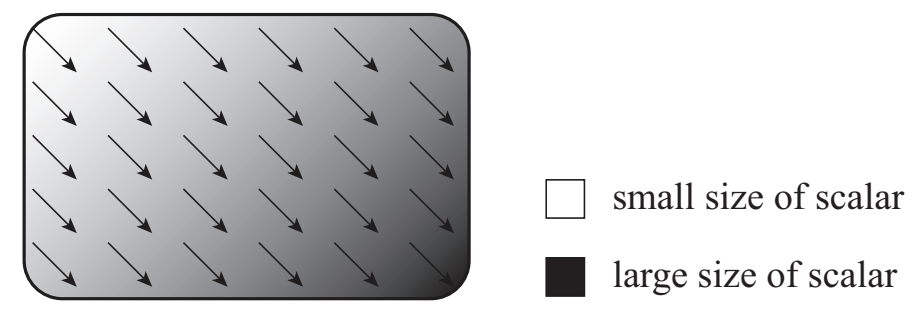

FIG. 2: Lorentz breakdown through spacetime-dependent scalars. The background shading of gray measures the size of the scalar: the lighter regions correspond to smaller values of the scalar. The black arrows represent the gradient, which selects a preferred direction in the vacuum. It follows that Lorentz symmetry is broken.

\section{THE STANDARD-MODEL EXTENSION}

In order to establish the low-energy phenomenology of Lorentz and CPT breaking and to identify relevant experimental signals for these effects, a suitable test framework is desirable. A number of Lorentz-symmetry tests are motivated and analyzed in purely kinematical models that describe small deviations from Lorentz invariance, such as Robertson's framework and its Mansouri-Sexl extension, the $c^{2}$ model, and phenomenologically constructed modified one-particle dispersion relations. However, the CPT properties of these test models lack clarity, and the absence of dynamical features greatly restricts their scope. To circumvent these issues, the SME, already mentioned in the introduction, has been developed. The present section contains a brief review of the philosophy behind the construction of the SME.

Let us first argue in favor of a dynamical rather than a purely kinematical test model. When the kinematical rules are fixed, there is certainly some residual freedom in introducing corresponding dynamical features. However, the dynamics is constrained by the requirement that established physics must be recovered in certain limits. Moreover, it seems complicated and may not even be possible to construct an effective theory that contains the Standard Model with dynamics considerably different from that of the SME. We also mention that kinematical investigations are limited to only a subset of potential Lorentz-violation signals emerging from fundamental physics. From this point of view, it appears to be desirable to implement explicitly dynamical features of sufficient generality into test frameworks for Lorentz and CPT invariance.

The generality of the SME. In order to recognize the generality of the SME, we review the main ingredients of its construction [10, 11. Starting from the conventional Standard-Model and general-relativity Lagrangians $\mathcal{L}_{\mathrm{SM}}$ and $\mathcal{L}_{\text {gr }}$, respectively, Lorentz-violating corrections $\delta \mathcal{L}$ are added:

$$
\mathcal{L}_{\mathrm{SME}}=\mathcal{L}_{\mathrm{SM}}+\mathcal{L}_{\mathrm{gr}}+\delta \mathcal{L}
$$

Here, $\mathcal{L}_{\mathrm{SME}}$ denotes the SME Lagrangian. The correction terms $\delta \mathcal{L}$ are formed by contracting Standard-Model and gravitational fields of any mass dimensionality with Lorentz-breaking tensorial coefficients that describe a nontrivial vacuum with background vectors or tensors. This background is presumed to originate from effects in the underlying theory, such as those discussed in the previous section. To ensure coordinate independence, these contractions must yield coordinate Lorentz scalars. We remark that in a curved-background context involving gravity, this procedure is most easily implemented employing the vierbein. It thus becomes clear that all possible contributions to $\delta \mathcal{L}$ determine the most general effective dynamical description of first-order Lorentz violation at the level of observer Lorentz-invariant unitary effective field theory.

Other potential features of underlying physics, such as non-pointlike elementary particles or a discrete spacetime structure at the Planck length, are not likely to invalidate this effective-field-theory approach at presently attainable energies. On the contrary, the phenomenologically successful Standard Model and general relativity are widely believed to be effective-field-theory limits of more fundamental physics. If underlying physics indeed leads to minuscule Lorentzbreaking effects, it would appear somewhat artificial to consider low-energy effective models outside the framework of effective quantum field theory. We finally note that the requirement for a low-energy description beyond effective field theory is also unlikely to arise within the context of underlying physics with novel Lorentz-symmetric features, such as additional particles, new symmetries, or large extra dimensions. Note in particular that Lorentz-invariant modifications can therefore easily be implemented into the SME, should it become necessary [12].

Advantages of the SME. The SME allows the identification and direct comparison of virtually all presently feasible experiments that search for deviations from Lorentz and CPT symmetry. Moreover, certain limits of the SME correspond to classical kinematics test models of relativity theory (such as the previously mentioned frame- 
work by Robertson, its Mansouri-Sexl extension to arbitrary clock synchronizations, or the $c^{2}$ model) [11, 13. A further benefit of the SME is the possibility of implementing additional desirable features besides coordinate independence. For example, one can choose to impose spacetime-translation invariance (at least in the flat-spacetime limit), $\mathrm{SU}(3) \times \mathrm{SU}(2) \times \mathrm{U}(1)$ gauge invariance, power-counting renormalizability, hermiticity, and local interactions. These requirements place additional constraints on the parameter space for Lorentz and CPT breakdown. Another possibility is to make simplifying choices, such as a residual rotational symmetry in certain inertial frames. This latter hypothesis together with additional simplifications of the SME has been adopted in some investigations [14].

\section{LORENTZ AND CPT TESTS}

The full SME contains an infinite number of Lorentz- and CPT-violating coefficients. However, in an effective field theory one might generically expect the power-counting renormalizable operators to dominate at low energies. The restriction to this subset of the SME is called the minimal Standard-Model Extension (mSME). To date, the flat-spacetime limit of the mSME has been the basis for numerous phenomenological investigations of Lorentz and CPT violation in many physical systems including mesons [15] 21], baryons [22 24], electrons [25] 27, photons [13, 28], muons [29], and the Higgs sector [30. Studies involving the curved-spacetime sector of the mSME have recently also been performed [31. We note that neutrino-oscillation measurements harbor the potential for discovery [10, 32, 33]. This section contains a brief description of a representative sample of experimental efforts.

Tests involving particle collisions. One of the key predictions of the mSME is that one-particle dispersion relations are typically modified by Lorentz and CPT violation. These modifications would result in changes to the kinematics of particle-collision processes. (Energy-momentum remains conserved in the context of the flat-spacetime mSME because the Lorentz- and CPT-violating coefficients are taken as spacetime constant.) For example, reaction thresholds may be shifted, reactions kinematically forbidden in Lorentz-symmetric physics may now occur, and certain conventional reactions may no longer be allowed kinematically.

Consider, for example, the spontaneous emission of a photon from a free electron. In conventional physics, energymomentum conservation does not allow this process to occur. However, certain types of Lorentz and CPT breakdown can slow down light relative to the speed of electrons. In analogy to the usual Cherenkov effect (when light travels slower inside a macroscopic medium with refractive index $n>1$ ), electrons can then emit Cherenkov photons in such a Lorentz- and CPT-violating vacuum [14, 35. This "vacuum Cherenkov radiation" may or may not be a threshold effect depending on the type of mSME coefficient. Let us consider mSME coefficients that are associated with a threshold for the vacuum Cherenkov effect. In such a situation, we obtain an observational constraint on the size of these mSME coefficients as follows. Electrons traveling with a speed above the modified light speed cannot do so for long: they would slow down below threshold through the emission of vacuum Cherenkov radiation. It follows that if highly energetic stable electrons exist in nature, they must be below threshold. From this information one can extract a lower bound for the threshold, which in turn gives a constraint on Lorentz breaking. Employing LEP electrons with energies up to $104.5 \mathrm{GeV}$ in this context yields the limit $\tilde{\kappa}_{\text {tr }}-\frac{3}{4} c^{00} \lesssim 1.2 \times 10^{-11}[36$.

Next, consider photon decay in vacuum. This is another particle reaction process not allowed by energy-momentum conservation in ordinary physics. However, in the presence of certain mSME coefficients, light may travel faster than the maximal attainable speed of electrons. In analogy to the above vacuum-Cherenkov case, in which high-energy electrons become unstable, we expect that high-energy photons can now decay into an electron-positron pair. With the modified dispersion relations predicted by the mSME, one can indeed verify that this expectation is met. As for vacuum Cherenkov radiation, photon decay in a Lorentz-violating vacuum is often a threshold effect and can then be employed to extract an observational limit on this particular type of Lorentz breakdown. The idea is the following. If stable photons are observed, they must essentially be below the decay threshold. It then follows that the threshold energy must be higher than the energy of these stable photons. This constraint on the threshold energy results in a limit on the size of the corresponding type of Lorentz violation. At the Tevatron, stable photons with energies up to $300 \mathrm{GeV}$ were observed. In this situation, our reasoning gives the bound $-5.8 \times 10^{-12} \lesssim \tilde{\kappa}_{\mathrm{tr}}-\frac{3}{4} c^{00}[36$.

We note that the above results assume that both vacuum Cherenkov radiation and photon decay are efficient enough. The purely kinematical arguments we have presented are insufficient for conservative observational limits. This is consistent with our remarks in the previous section that a dynamical framework is desirable, and the full mSME (not only the predicted modified dispersion relations) are needed. Appropriate calculations within the mSME indeed establish that the rates for vacuum Cherenkov radiation and photon decay would be fast enough to validate the above reasoning [36, 37.

Spectropolarimetry of cosmological sources. The pure electrodynamics sector of the mSME contains one type of coefficient that violates both Lorentz and CPT invariance. It is a mass dimension three term of Chern-Simons type parametrized by the $\left(k_{A F}\right)^{\mu}$ background 4-vector. Among other effects, the $\left(k_{A F}\right)$ term results in birefringence for photons [38, the vacuum Cherenkov effect [35, as well as shifts in cavity frequencies [39]. These deviations 


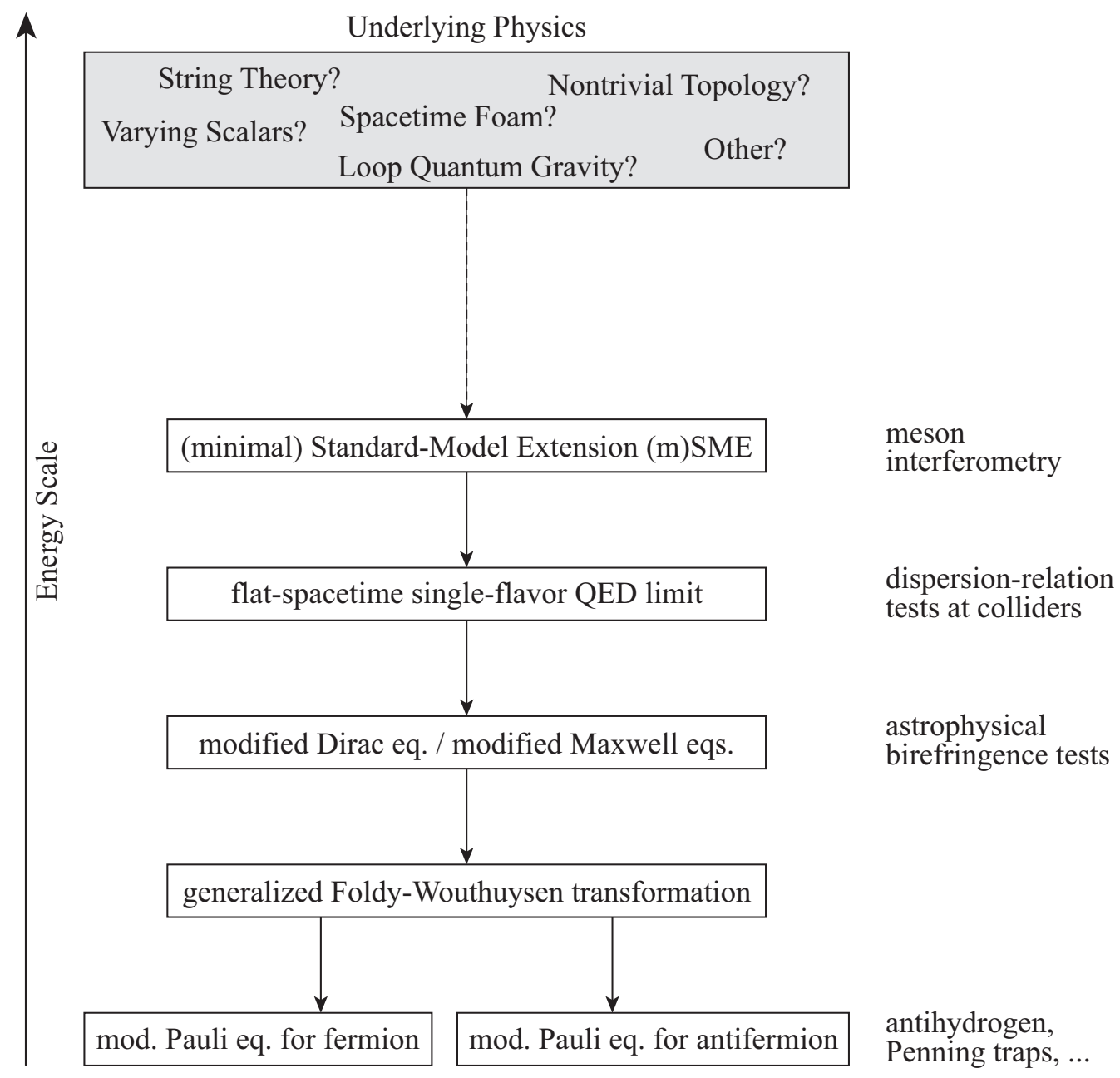

FIG. 3: Flow chart for phenomenological SME analyses. The neutral-meson effective Hamiltonian is derived from the quark sector of the mSME. Collider tests involve photon decay, which requires a tree-level QFT calculation. Spectropolarimetric studies of astrophysical sources are performed within the context of the classical (modified) Maxwell equations. CPT violation in the mSME leads to different Pauli-type equations for a fermion and its antifermion. These modified Pauli equations are employed for calculations of atomic spectra, for example.

from established physics are accessible to experimental investigations. Birefringence searches in cosmic radiation are particularly well suited since the extremely long propagation time is directly associated with an ultrahigh sensitivity to this type of Lorentz and CPT breakdown. Spectropolarimetric studies of experimental data from cosmological sources have established a limit on $\left(k_{A F}\right)^{\mu}$ at the level of $10^{-42} \mathrm{GeV} 38$.

Investigations of cold antihydrogen. A comparison of the spectra of hydrogen $(\mathrm{H})$ and antihydrogen $(\overline{\mathrm{H}})$ is well suited for Lorentz- and CPT-violation searches. There are a number of transitions that one can consider. One of them, the unmixed $1 \mathrm{~S}-2 \mathrm{~S}$ transition, seems to be an exquisite candidate: its projected experimental sensitivity is anticipated to be roughly at the level of $10^{-18}$, which is auspicious in light of the expected Planck-scale suppression of quantum-gravity effects. However, a leading-order calculation within the mSME predicts identical shifts for free $\mathrm{H}$ or $\overline{\mathrm{H}}$ in the initial and final levels with respect to the conventional energy states. From this point of view, the $1 \mathrm{~S}-2 \mathrm{~S}$ transition is actually less satisfactory for the determination of unsuppressed Lorentz- and CPT-breaking effects. Within the mSME, the leading non-trivial contribution to this transition is generated by relativistic corrections, and it comes with two additional powers of the fine-structure constant $\alpha$. The predicted shift in the transition frequency, already expected to be minute at zeroth order in $\alpha$, is thus associated with a further suppression factor of more than ten thousand [40].

An additional transition that can be used for Lorentz and CPT tests is the spin-mixed $1 \mathrm{~S}-2 \mathrm{~S}$ transition. When $\mathrm{H}$ or $\overline{\mathrm{H}}$ is trapped with electromagnetic fields (e.g., in a Ioffe-Pritchard trap) the $1 \mathrm{~S}$ and the $2 \mathrm{~S}$ states are each split as a result of the usual Zeeman effect. The mSME then predicts that in this case the $1 \mathrm{~S}-2 \mathrm{~S}$ transition between the spin-mixed states is indeed affected by Lorentz- and CPT-violating coefficients at leading order. A disadvantage 
from a practical perspective is the magentic-field dependence of this transition, so that the experimental sensitivity is limited by the size of the inhomogeneity of the $\vec{B}$ field in the trap. The development of new experimental techniques might avoid this issue, and a frequency resolutions close to the natural linewidth could then be attained [40.

A third transition interesting from a Lorentz- and CPT-violation perspective is the hyperfine Zeeman transitions within the 1S state itself. Even in the limit of a zero $\vec{B}$ field, the mSME establishes leading-order level shifts for two of the transitions between the Zeeman-split states. We remark that this result may also be advantageous from an experimental perspective because a number of other transitions of this type, such as the conventional H-maser line, can be well resolved in the laboratory [40].

Tests in Penning traps. The mSME establishes not only that atomic energy levels can be affected by the presence of Lorentz and CPT breakdown, but also, for instance, the levels of protons and antiprotons inside a Penning trap. A perturbative calculation predicts that only one mSME coefficient (a CPT-violating $b^{\mu}$-type background vector, which is coupled to the chiral current of a fermion) affects the transition-frequency shifts between the proton and its antiparticle at leading order. To be more specific, the anomaly frequencies are displaced in opposite directions for protons and antiprotons. This effect can be used to determine a clean experimental constraint on the proton's $b^{\mu}$ coefficient [25].

Neutral-meson interferometry. A long established standard CPT-symmetry test is the comparison of the Kmeson's mass to that of the corresponding antimeson: even extremely small mass differences would yield measurable effects in Kaon-interferometry experiments. In spite of the fact that the mSME contains only one mass operator for a given quark species and the associated antiquark species, these (anti)particles are nevertheless influenced differently by the Lorentz- and CPT-breaking background in the mSME. This causes the dispersion relations for a meson and its antimeson to differ, so that mesons and antimesons can possess distinct energies despite having equal 3-momenta. It is this split in energy that is ultimately measurable in interferometric experiments, and it is thus potentially observable in such systems. We remark that not only the K-meson but also other neutral mesons can be investigated. Note in particular that in addition to CPT breakdown, Lorentz violation is involved as well, so that boost- and rotation-dependent effects can be looked for [15,21].

\section{SUMMARY}

To date, no credible observational evidence for deviations from relativity theory exist. However, in theoretical approaches to underlying physics, such as in models of the Planck-length structure of spacetime, minuscule violations of Lorentz and translation symmetry can be accommodated. In this mini course, we have given an overview of the motivations, theoretical ideas, and experimental efforts in this spacetime-symmetry-breaking context.

We have argued that quantum-gravity models, for example, should describe a quantized version of the dynamics of spacetime. In such a quantized spacetime, the concept of a smooth manifold may break down at some small distance scale, so that the usual spacetime symmetries may only emerge at low energies. We have reviewed two specific examples how Lorentz invariance might be violated: in string field theory (i.e., via spontaneous symmetry breaking) or in the context of varying scalars (i.e., via the gradient of the scalar).

At presently attainable energies and under mild assumptions regarding the dynamics, the effects of general Lorentz and CPT breakdown are described by an effective field theory called the SME. This framework contains essentially the entire body of established physics (i.e., the Standard model and general relativity), so that predictions for Lorentzand CPT-breaking effects in essentially all physical systems are possible, at least in principle. The coefficients for Lorentz and CPT violation in the SME are prescribed non-dynamical background vectors and tensors assumed to be generated by more fundamental physics.

Spacetime symmetries underpin numerous physical effects. Accordingly, Lorentz and CPT invariance can be tested in a wide variety of physical systems. This fact, together with the generality of the SME and the strong motivations for Lorentz and CPT violations, has led to a recent surge of experimental efforts to test relativity theory. We have reviewed a representative sample of these efforts in the contexts of dispersion-relation studies, astrophysical polarimetry, and matter-antimatter comparisons.

A variety of important unanswered questions remain in this field. They are of theoretical, of phenomenological, as well as of experimental nature, and they provide ample ground for further research in spacetime-symmetry physics. 


\section{Acknowledgments}

The author wishes to thank the organizers for the invitation to present this mini course. This work was funded in part by CONACyT under Grant No. 55310.

[1] See, e.g., R.G. Sachs, The Physics of Time Reversal, University of Chicago Press, Chicago, 1987.

[2] V.A. Kostelecký and S. Samuel, Phys. Rev. D 39, 683 (1989); Phys. Rev. Lett. 63, 224 (1989); 66, 1811 (1991); V.A. Kostelecký and R. Potting, Nucl. Phys. B 359, 545 (1991); Phys. Lett. B 381, 89 (1996); Phys. Rev. D 63, 046007 (2001); V.A. Kostelecký et al., Phys. Rev. Lett. 84, 4541 (2000).

[3] J. Alfaro, H.A. Morales-Técotl, and L.F. Urrutia, Phys. Rev. Lett. 84, 2318 (2000); Phys. Rev. D 65, 103509 (2002); D. Sudarsky et al., Phys. Rev. D 68, 024010 (2003).

[4] F.R. Klinkhamer, Nucl. Phys. B 578, 277 (2000).

[5] V.A. Kostelecký et al., Phys. Rev. D 68, 123511 (2003); O. Bertolami et al., Phys. Rev. D 69, 083513 (2004).

[6] C. Amsler et al. [Particle Data Group], Phys. Lett. B 667, 1 (2008).

[7] O.W. Greenberg, Phys. Rev. Lett. 89, 231602 (2002).

[8] For a somewhat more pedestrian exposition, see O.W. Greenberg, Found. Phys. 36, 1535 (2006).

[9] See, e.g., N.E. Mavromatos, A. Meregaglia, A. Rubbia, A. Sakharov, and S. Sarkar, Phys. Rev. D 77, 053014 (2008).

[10] D. Colladay and V.A. Kostelecký, Phys. Rev. D 55, 6760 (1997); 58, 116002 (1998); V.A. Kostelecký and R. Lehnert, Phys. Rev. D 63, 065008 (2001); V.A. Kostelecký, Phys. Rev. D 69, 105009 (2004); R. Bluhm and V.A. Kostelecký, Phys. Rev. D 71, 065008 (2005).

[11] V.A. Kostelecký and M. Mewes, Phys. Rev. D 80, 015020 (2009).

[12] M.S. Berger and V.A. Kostelecký, Phys. Rev. D 65, 091701(R) (2002); H. Belich et al., Phys. Rev. D 68, 065030 (2003); M.S. Berger, Phys. Rev. D 68, 115005 (2003).

[13] V.A. Kostelecký and M. Mewes, Phys. Rev. D 66, 056005 (2002).

[14] S. Coleman and S.L. Glashow, Phys. Rev. D 59, 116008 (1999).

[15] KTeV Collaboration, H. Nguyen, arXiv:hep-ex/0112046 Y.B. Hsiung et al., Nucl. Phys. Proc. Suppl. 86, 312 (2000).

[16] FOCUS Collaboration, J.M. Link et al., Phys. Lett. B 556, 7 (2003).

[17] OPAL Collaboration, R. Ackerstaff et al., Z. Phys. C 76, 401 (1997); DELPHI Collaboration, M. Feindt et al., preprint DELPHI 97-98 CONF 80 (1997); BELLE Collaboration, K. Abe et al., Phys. Rev. Lett. 86, 3228 (2001); BaBar Collaboration, B. Aubert et al., Phys. Rev. Lett. 92, 181801 (2004).

[18] BaBar Collaboration, B. Aubert et al., arXiv:hep-ex/0607103 G. Amelino-Camelia et al., Eur. Phys. J. C 68, 619 (2010).

[19] V.A. Kostelecký and R. Potting, Phys. Rev. D 51, 3923 (1995).

[20] D. Colladay and V.A. Kostelecký, Phys. Lett. B 344, 259 (1995); Phys. Rev. D 52, 6224 (1995); V.A. Kostelecký and R. Van Kooten, Phys. Rev. D 54, 5585 (1996); O. Bertolami et al., Phys. Lett. B 395, 178 (1997); N. Isgur et al., Phys. Lett. B 515, 333 (2001).

[21] V.A. Kostelecký, Phys. Rev. Lett. 80, 1818 (1998); Phys. Rev. D 61, 016002 (2000); Phys. Rev. D 64, 076001 (2001).

[22] D. Bear et al., Phys. Rev. Lett. 85, 5038 (2000); D.F. Phillips et al., Phys. Rev. A 62, 063405 (2000); Phys. Rev. D 63, 111101 (2001); M.A. Humphrey et al., Phys. Rev. A 68, 063807 (2003); V.A. Kostelecký and C.D. Lane, Phys. Rev. D 60, 116010 (1999); J. Math. Phys. 40, 6245 (1999); I. Altarev et al., Phys. Rev. Lett. 103, 081602 (2009); Europhys. Lett. 92, $51001(2010)$.

[23] R. Bluhm et al., Phys. Rev. Lett. 88, 090801 (2002); Phys. Rev. D 68, 125008 (2003); R. Lehnert, Phys. Rev. D 68, 085003 (2003).

[24] F. Canè et al., Phys. Rev. Lett. 93, 230801 (2004); P. Wolf et al., Phys. Rev. Lett. 96, 060801 (2006).

[25] H. Dehmelt et al., Phys. Rev. Lett. 83, 4694 (1999); R. Mittleman et al., Phys. Rev. Lett. 83, 2116 (1999); G. Gabrielse et al., Phys. Rev. Lett. 82, 3198 (1999); Phys. Rev. Lett. 79, 1432 (1997); Phys. Rev. D 57, 3932 (1998); C.D. Lane, Phys. Rev. D 72, 016005 (2005).

[26] L.-S. Hou et al., Phys. Rev. Lett. 90, 201101 (2003); R. Bluhm and V.A. Kostelecký, Phys. Rev. Lett. 84, 1381 (2000); B.R. Heckel et al., Phys. Rev. Lett. 97, 021603 (2006).

[27] H. Müller et al., Phys. Rev. D 68, 116006 (2003); H. Müller, Phys. Rev. D 71, 045004 (2005); B. Altschul, Phys. Rev. Lett. 96, 201101 (2006); Phys. Rev. D 80, 091901 (2009) Phys. Rev. D 81, 041701 (2010).

[28] V.A. Kostelecký and M. Mewes, Phys. Rev. Lett. 87, 251304 (2001); J. Lipa et al., Phys. Rev. Lett. 90, 060403 (2003); Q. Bailey and V.A. Kostelecký, Phys. Rev. D 70, 076006 (2004); B. Feng et al., Phys. Rev. Lett. 96, 221302 (2006); V.A. Kostelecký and M. Mewes, Phys. Rev. Lett. 97, 140401 (2006); B. Altschul, Phys. Rev. Lett. 98, 041603 (2007); C.D. Carone, M. Sher, and M. Vanderhaeghen, Phys. Rev. D 74, 077901 (2006):

[29] V.W. Hughes et al., Phys. Rev. Lett. 87, 111804 (2001); G.W. Bennett et al. [Muon (g-2) Collaboration], Phys. Rev. Lett. 100, 091602 (2008); R. Bluhm et al., Phys. Rev. Lett. 84, 1098 (2000).

[30] D.L. Anderson et al., Phys. Rev. D 70, 016001 (2004).

[31] J.B.R. Battat, J.F. Chandler, and C.W. Stubbs, Phys. Rev. Lett. 99, 241103 (2007); H. Müller et al., Phys. Rev. Lett. 100, 031101 (2008); K.Y. Chung et al., Phys. Rev. D 80, 016002 (2009); Q.G. Bailey and V.A. Kostelecký, Phys. Rev. D 74, 045001 (2006); V.A. Kostelecký, N. Russell, and J. Tasson, Phys. Rev. Lett. 100, 111102 (2008); Q.G. Bailey, Phys. 
Rev. D 80, 044004 (2009); V.A. Kostelecký and J. Tasson, Phys. Rev. Lett. 102, 010402 (2009).

[32] V.D. Barger et al., Phys. Rev. Lett. 85, 5055 (2000); J.N. Bahcall et al., Phys. Lett. B 534, 120 (2002); V.A. Kostelecký and M. Mewes, Phys. Rev. D 70, 031902 (2004); Phys. Rev. D 70, 076002 (2004); T. Katori, V.A. Kostelecký, and R. Tayloe, Phys. Rev. D 74, 105009 (2006); J.S. Díaz, V.A. Kostelecký, and M. Mewes, Phys. Rev. D 80, 076007 (2009); S. Hollenberg, O. Micu, and H. Päs, Phys. Rev. D 80, 053010 (2009); J.S. Díaz and A. Kostelecký, arXiv:1012.5985 [hep-ph].

[33] V.A. Kostelecký and M. Mewes, Phys. Rev. D 69, 016005 (2004).

[34] R. Lehnert, J. Math. Phys. 45, 3399 (2004); B. Altschul and V.A. Kostelecký, Phys. Lett. B 628, 106 (2005); J. Alfaro et al., Phys. Lett. B 639, 586 (2006); R. Lehnert, Phys. Rev. D 74, 125001 (2006); A.J. Hariton and R. Lehnert, Phys. Lett. A 367, 11 (2007); R. Lehnert, Rev. Mex. Fís. 56 (6), 469 (2010); C.M. Reyes, L.F. Urrutia, and J.D. Vergara, Phys. Rev. D 78, 125011 (2008); Phys. Lett. B 675, 336 (2009); C. Armendariz-Picon and A. Diez-Tejedor, JCAP 0912, 018 (2009); C.M. Reyes, Phys. Rev. D 80, 105008 (2009); Phys. Rev. D 82, 125036 (2010); J. Alfaro et al., Int. J. Mod. Phys. A 25, 3271 (2010); J. Alfaro and L.F. Urrutia, Phys. Rev. D 81, 025007 (2010); B. Altschul, Q.G. Bailey, and V.A. Kostelecký, Phys. Rev. D 81, 065028 (2010); C. Armendariz-Picon et al., JHEP 1010, 079 (2010); M. Cambiaso and L.F. Urrutia, Phys. Rev. D 82, 101502 (2010); R. Casana et al., Phys. Rev. D 80, 125040 (2009); Phys. Rev. D 82, 125006 (2010).

[35] R. Lehnert and R. Potting, Phys. Rev. Lett. 93, 110402 (2004); Phys. Rev. D 70, 125010 (2004); K.G. Zloshchastiev, arXiv:1003.0657 [hep-th].

[36] M.A. Hohensee et al., Phys. Rev. Lett. 102, 170402 (2009); Phys. Rev. D 80, 036010 (2009); See also J.-P. Bocquet et al. [GRAAL Collaboration], Phys. Rev. Lett. 104, 241601 (2010).

[37] F.R. Klinkhamer and M. Schreck, Phys. Rev. D 78, 085026 (2008).

[38] S.M. Carroll, G.B. Field, and R. Jackiw, Phys. Rev. D 41, 1231 (1990).

[39] M. Mewes, Phys. Rev. D 78, 096008 (2008).

[40] R. Bluhm, V.A. Kostelecký, and N. Russell, Phys. Rev. Lett. 82, 2254 (1999). 